\title{
MicroRNA-505 functions as a tumor suppressor in endometrial cancer by targeting TGF- $a$
}

\author{
Shuo Chen ${ }^{1}$, Kai-Xuan Sun ${ }^{1}$, Bo-Liang Liu', Zhi-Hong Zong ${ }^{2}$ and Yang Zhao ${ }^{{ }^{*}}$
}

\begin{abstract}
Background: Endometrial carcinoma (EC) is one of the most lethal gynecologic cancers. Patients frequently have regional or distant metastasis at diagnosis. MicroRNAs are small non-coding RNAs that participate in numerous biological processes. Recent studies have demonstrated that miR-505 is associated with several types of cancer; however, the expression and function of miR-505 have not been investigated in EC.

Methods: miR-505 expression in normal endometrial tissue, endometrial carcinomas were quantified by Quantitative reverse transcription PCR. The endometrial carcinoma cell lines HEC-1B and Ishikawa were each transfected with miR-505 or scrambled mimics, after which cell phenotype and expression of relevant molecules were assayed. Dual-luciferase reporter assay and a xenograft mouse model were used to examine miR-505 and its target gene TGF-a.

Results: RT-PCR results demonstrated that miR-505 was significantly downregulated in human EC tissues compared to normal endometrial tissues. Besides, miR-505 expression was negatively associated with FIGO stage (stage I-II vs. III-IV), and lymph node metastasis (negative vs. positive). In vitro, overexpression of miR-505 significantly suppressed EC cell proliferation, increased apoptosis and reduced migratory and invasive activity. A miR-505 binding site was identified in the 3' untranslated region of TGF-a mRNA (TGFA) using miRNA target-detecting software; a dual luciferase reporter assay confirmed that miR-505 directly targets and regulates TGFA. RT-PCR and Western-blotting results indicated that overexpressing miR-505 reduced the expression of TGF- $a$ and the TGF-a-regulated proteins MMP2, MMP9, CDK2, while induced Bax and cleaved-PARP expression in EC cells. In vivo, overexpression of miR-505 reduced the tumorigenicity and inhibited the growth of xenograft tumors in a mouse model of EC.
\end{abstract}

Conclusions: Taken together, this study demonstrates that miR-505 acts as tumor suppressor in EC by regulating TGF-a.

Keywords: MiR-505, TGF-a, Endometrial carcinoma, Tumorigenesis, Progression

\section{Background}

Endometrial carcinoma (EC) is one of the three most common types of gynecologic cancer and its global incidence has increased in recent years [1]. It is known that endometrial carcinoma is divided into estrogen dependent (type I) and non-estrogen dependent (type II) $[2,3]$. The pathogenesis of type I endometrial carcinoma is that under the long-term effects of estrogen, but without the antagonistic effects of progesterone, the endometrium

\footnotetext{
* Correspondence: yida.zhaoyang@163.com

${ }^{1}$ Department of Gynecology, The First Affiliated Hospital of China Medical University, Shenyang 110001, China

Full list of author information is available at the end of the article
}

progressively increases in thickness and therefore becomes more susceptible to becoming cancerous $[4,5]$. Generally, FIGO stage I (2009) endometrial carcinoma is considered to have a good prognosis with a 5-year survival rate of up to $96 \%$ [6]. Unfortunately, as multiple factors affect recurrence, including surgical stage, differentiation and lymph node metastasis, effective therapies for patients with advanced-stage EC or disease recurrence are still lacking [7], the 5-year OS rate of the FIGO stages II-IV was $76.0 \%$ [8]. The identification and further elucidation of the molecular mechanisms responsible for EC tumorigenesis and progression may have a major impact on the health of females. 
MicroRNAs are a group of endogenous, small, 18-25 base-long non-coding RNAs that influence multiple biological processes including proliferation, apoptosis, senescence, differentiation and development by posttranscriptionally repressing gene expression or inducing mRNA degradation via binding to the 3 ' untranslated regions (UTR) of specific target genes [9-11]. MicroRNAs can target approximately $20-30 \%$ of genes. A single microRNA can target at least 200 genes, and a single gene can be regulated by many RNAs [12-14]. A large body of research has demonstrated that dysregulation of microRNAs can promote tumorigenesis and metastasis $[15,16]$. Recent studies have demonstrated that microRNAs can act as either potent oncogenes or tumor suppressor genes [17].

MiR-505 has been identified to function as a tumor suppressor in breast cancer [18-20]. Numerous studies have indicated that miR-505 inhibits cell proliferation by inducing apoptosis and can promote chemoresistance in breast cancer [18, 21]. Expression of miR-505 correlates with established prognostic biomarkers in breast cancer [22]. Moreover, data from mouse models has indicated an association between miR-505 and human basal-type breast cancer [19]. Another study recently provided evidence that miR-505 could suppress the epithelialmesenchymal transition (EMT) and metastasis in nasopharyngeal carcinoma [23]. However, little is known about the biological function and target genes of miR-505 in EC.

Transforming growth factor- $\alpha$ (TGF- $\alpha$ ) is a member of the epidermal growth factor family of mitogens and is encoded by the TGFA gene. TGF- $\alpha$ binds to the epidermal growth factor receptor (EGFR) to initiate a series of biological processes including cell proliferation, differentiation and development [24]. TGF- $\alpha$ makes an important contribution to cell proliferation and invasion in triplenegative breast cancer [25], and strongly correlates with metastasis in advanced prostate cancer [26]. Besides, TGF- $\alpha$ is also involved in EC tumorigenesis and reported to promote angiogenesis in endothelial cells [27, 28]. Zhu et al. reported that miR-152 could potentially controls migration and invasion by targeting TGF- $\alpha$ in prostate cancer cell lines [29]; Jin et al. reported that miR-376c could inhibit cell proliferation and invasion by targeting TGF- $\alpha$ in osteosarcoma [30]; Qin et al. reported that miR-124 may regulates TGF- $\alpha$-induced EMT in human prostate cancer cells [31]. These studies suggest us that TGF-a may have potential as a target in inhibiting endometrial cancer tumorigenesis and progression through down-regulating by relative microRNA. We analyzed the mRNA sequence of TGF- $\alpha$ and identified binding sites for miR-505 in TGFA.

Here, we report that miR-505 functions as a tumor suppressor in EC by targeting and regulating TGFA.

\section{Results \\ Associations between miR-505 expression and the clinico- pathological features of EC}

Quantitative reverse transcription polymerase chain reaction (qRT-PCR) demonstrated that miR-505 was expressed at lower levels in human EC than normal endometrial tissues (Fig. 1a, b, $P<0.05$, Additional file 1: Table S1).

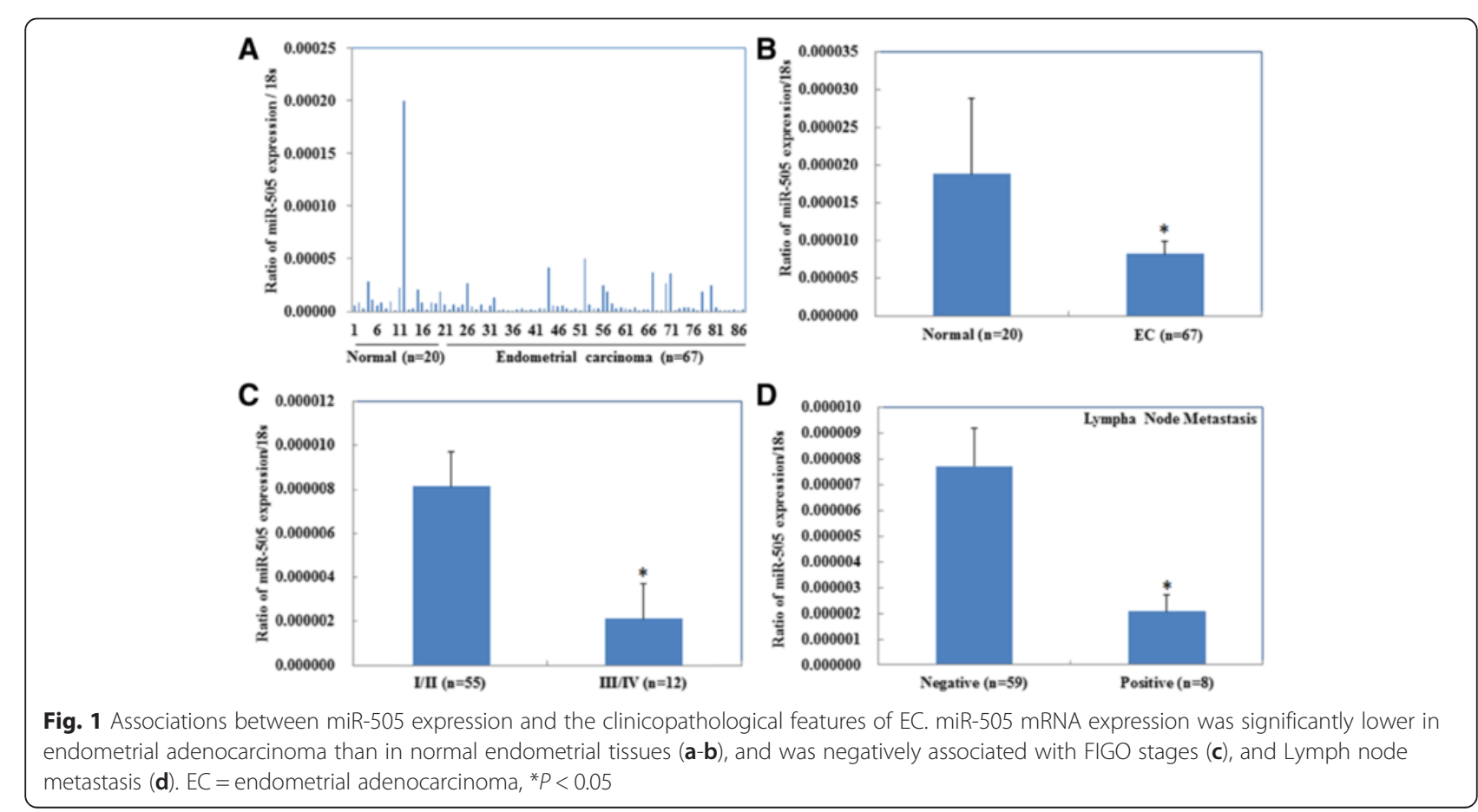


Besides, miR-505 expression was negatively associated with FIGO stage (Fig. 1c, stage I/II vs. stage III/IV, $P<0.05$ ), and lymph node metastasis (Fig. 1d, negative vs. positive, $P<$ $0.05)$. There were no significant associations between miR505 expression and the depth of invasion and pathological type (endometrial adenocarcinoma vs. other types, $P>$ 0.05 , Details could be found in Additional file 1: Table S2).

Overexpression of miR-505 inhibits proliferation, induces apoptosis and reduces the tumorigenicity of EC cells in vitro

Ishikawa and HEC-1B cell lines were transfected with miR-505 mimic or the scrambled mimic. The CCK-8 assay demonstrated that the miR-505 overexpression (Fig. 2a, $P<0.05$ ) significantly inhibited cell proliferation in both cell lines compared with the mock or negative control group (Fig. 2b, $P<0.05$ ). Furthermore, cytometry flow showed that overexpression of miR-505 induced significant G1 phase arrest (Fig. 3a, $P<0.05$; PI staining) and high levels of apoptosis (Fig. $3 \mathrm{~b}, P<0.05$, Annexin V-FITC and PI staining). Additionally, overexpression of miR-505 significantly reduced the invasion (Fig. 4a, $P<0.05$, Transwell assay) and migratory ability of both cell lines (Fig. 4b, $P<0.05$, wound healing assay). Details could be found in Additional file 1: Table S3.
miR-505 directly targets TGFA and regulates TGF- $a$ expression in EC cells

We analyzed the mRNA sequence of TGF- $\alpha$ using the miRNA target-detecting software and identified binding sites for miR-505 in TGFA (Fig. 5a). A dual-luciferase reporter assay indicated that miR-505 can bind to the 3' UTR of TGFA (Fig. 5b). Furthermore, RT-PCR and Western blotting demonstrated that overexpression of miR-505 downregulated the mRNA (Fig. 6a, $P<0.05$ ) or protein (Fig. $6 \mathrm{~b}, P<0.05$ ) expression of MMP2, MMP9, CDK2, and TGF- $\alpha$, while upregulated Bax and cleaved PARP expression in both cells.

Overexpression of miR-505 inhibits the growth of EC xenografts in a mouse model

We stably transfected HEC-1B cells with has-miR-505 or negative control vector (Mock) and established a mouse model of EC. Compared to the mock group, HEC-1B cells overexpressing miR-505 had a significantly lower tumor volume (Fig. $7 \mathrm{a}$ and $\mathrm{b}, P<0.05$ ) and slower rate of growth (Fig. $7 \mathrm{c}, P<0.05$ ). To determine whether miR-505 affected the expression of TGF- $\alpha$ in vivo, we performed immunohistochemical staining of the excised xenograft tumors. The tumors formed by the cells overexpressing miR-505 had a significantly lower TGF- $\alpha$ expression compared to the mock group (Fig. 8a and b).
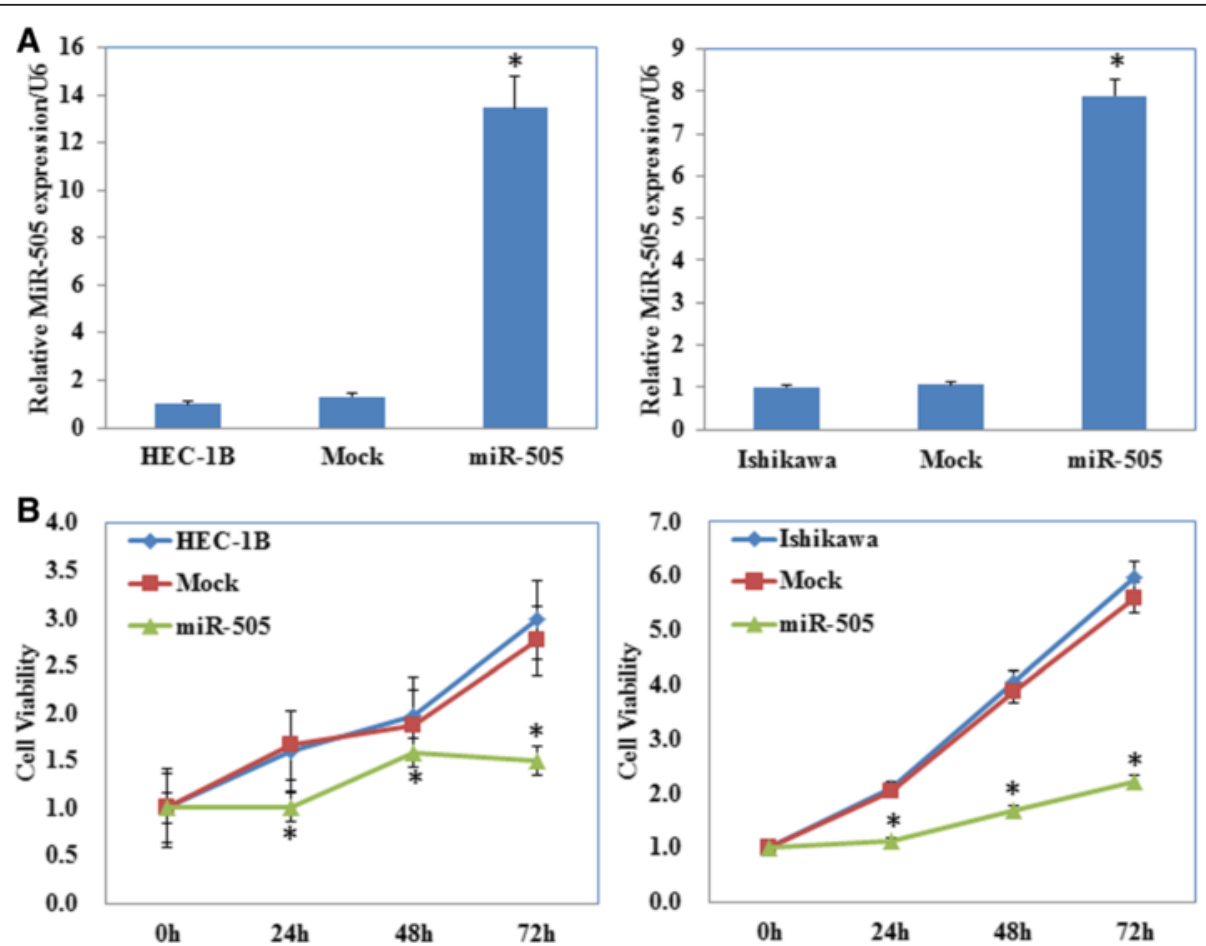

Fig. 2 miR-505 overexpression suppresses endometrial carcinoma cell proliferation in vitro. miR-505 transfection exhibited significantly higher miR-505 expression (a) and slower growth ability (b) compared with the control and mock cells. Results are representative of three separate experiments; data are expressed as the mean \pm standard deviation, ${ }^{*} P<0.05$ 


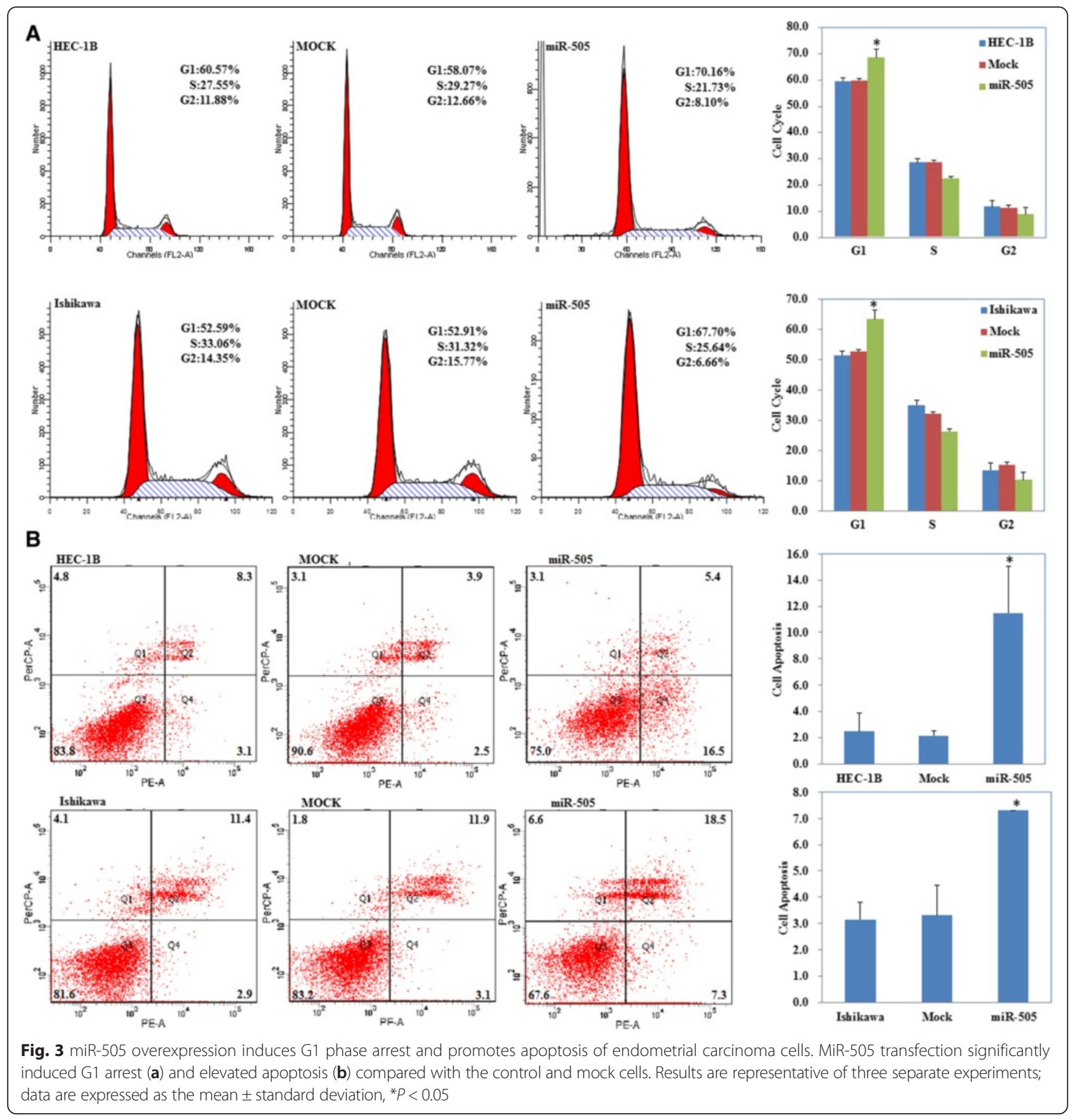

\section{Discussion}

This study demonstrates that miR-505 is significantly downregulated in human EC compared to normal endometrial tissues, and the expression of miR-505 was significantly associated with the FIGO stage and lymph node metastasis. Additionally, overexpression of miR505 significantly inhibited EC cell proliferation, induced G1-S arrest and apoptosis, and suppressed EC cell invasion and migration in vitro. Taken together, this data indicates that downregulation of miR-505 could promote the development and progression of EC.
The growth factor TGF- $\alpha$ is mainly secreted during embryogenesis and is frequently overexpressed in malignant tumors, including osteosarcoma, hepatic cancer, prostate cancer and breast cancer [20, 22, 26, 32, 33]. Recent studies have linked TGF- $\alpha$ overexpression to the invasive ability of triple-negative breast cancer cells [34]. Interestingly, overexpression of TGF- $\alpha$ could interact with mesenchymal-to-epithelial transition (MET) and contribute to cetuximab resistance in patients with colorectal cancer [35, 36]. High TGF- $\alpha$ expression was prognostic for poor overall survival in high-risk patients with melanoma 

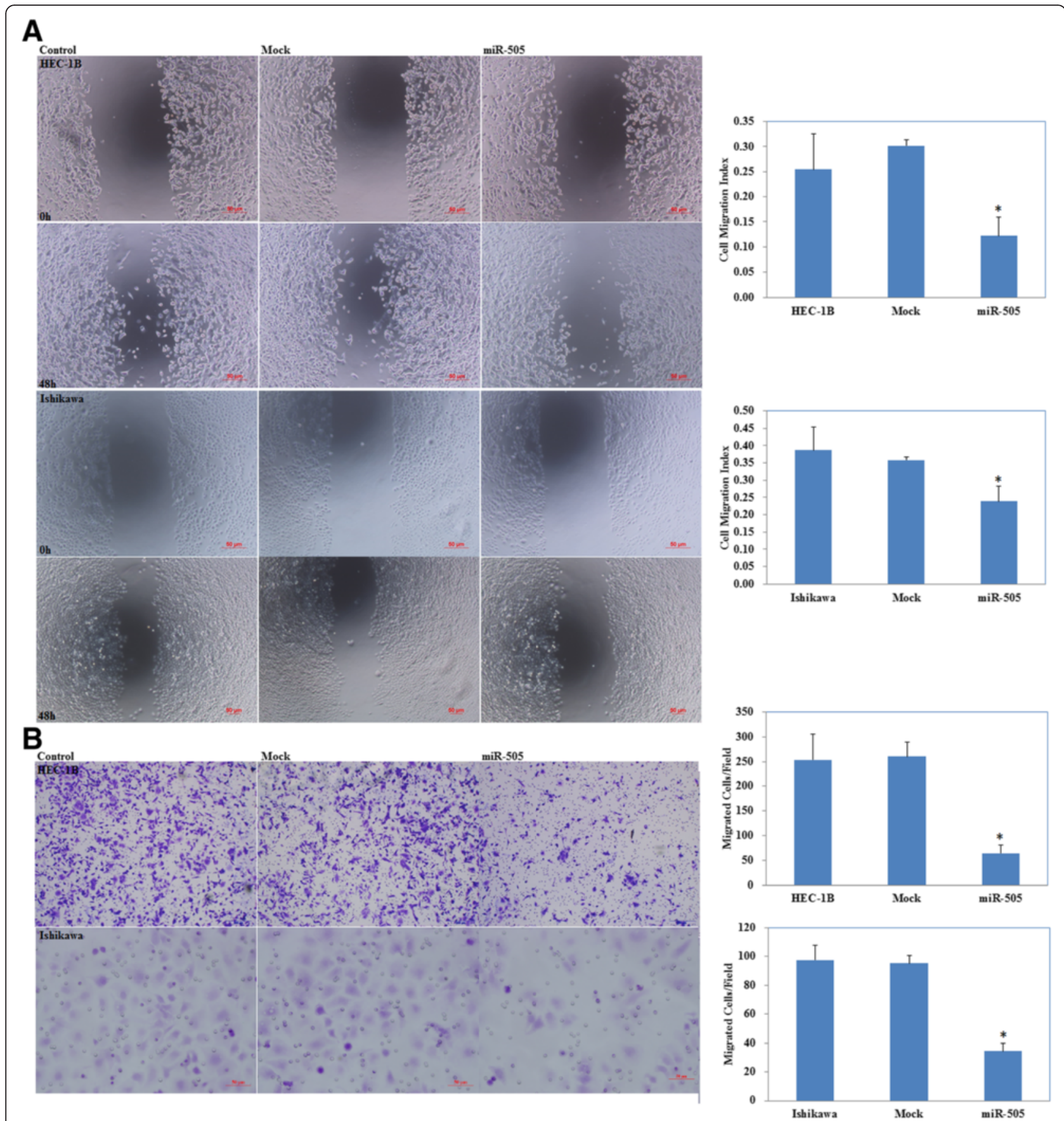

Fig. 4 Effects of miR-505 transfection on invasive and metastatic ability of endometrial adenocarcinoma cell lines in vitro. miR-505 overexpression reduced cell migration (a), and invasion ability (b) compared with the control and mock cells. Results are representative of three separate experiments; data are expressed as the mean \pm standard deviation, ${ }^{*} P<0.05$

[37]. In this study, target prediction software identified a miR-505 binding site in the 3' UTR of TGFA, and dualluciferase reporter assays confirmed that TGFA is a direct target of miR-505. In confirmation of this functional interaction, overexpression of miR-505 reduced both TGF- $\alpha$ mRNA and protein expression in EC cell lines.
TGF- $\alpha$ binds to the epidermal growth factor receptor (EGFR) to initiate multiple cellular events, including cell proliferation, and this process has been associated with tumorigenesis and angiogenesis [38, 39]. We infer that upregulation of miR-505 may inhibit proliferation, migration and invasion; promote apoptosis in EC by leading to 


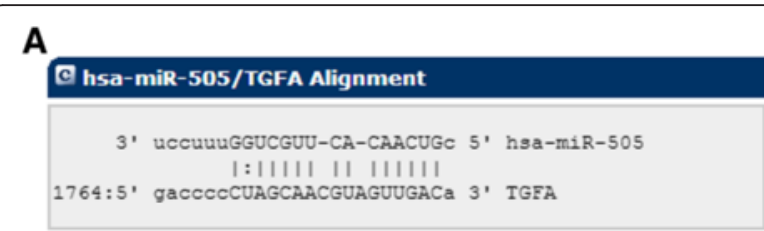

B

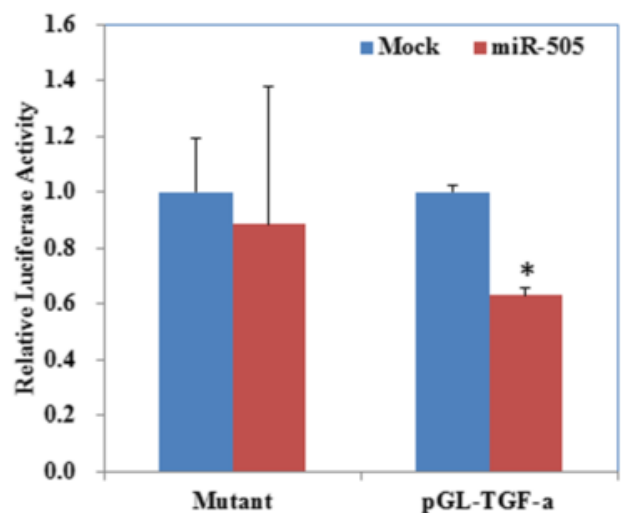

Fig. 5 miR-505 directly targets TGFA. The predicted seed region in the $3^{\prime}$ UTR of TGF- $a(\mathbf{a})$; Luciferase activity is unchanged when using a scrambled miRNA sequence or miR-505 with the mutant $3^{\prime}-U T R$ of TGF-a. However, when the wild-type 3'-UTR of TGF-a is used, the promoter activity is significantly reduced by miR-505 (b). Results are representative of three separate experiments; data are expressed as the mean \pm standard deviation, ${ }^{*} P<0.05$

decreased expression of TGF- $\alpha$. MMP2 and MMP9 are two of the most studied members of the matrix metalloproteinase (MMPs) family, and play central roles in cell invasion and metastasis by cleaving components of the extracellular matrix (ECM) [27]. In this study, overexpression of miR-505 downregulated both MMP2 and MMP9 mRNA and protein expression, providing further evidence that upregulation of miR-505 may inhibit invasion and metastasis in EC.

During late G1 phase, the CDK2/cyclin E complexes regulate the $\mathrm{G} 1$ to $\mathrm{S}$ phase transition and the $\mathrm{CDK} 2 /$ cyclin A complexes play an important role in $S$ phase progression $[40,41]$. Bcl-2 associated $\mathrm{X}$ protein (Bax) is a representative member of Bcl-2 family that promotes apoptosis via the caspase-dependent pathway and is frequently downregulated in cancer [42]. Poly ADP-ribose polymerase (PARP) participates in DNA repair and can inhibit apoptosis [43-45]. In this study, overexpression of miR505 reduced CDK2 mRNA and protein expression, upregulated Bax and increased the levels of cleaved PARP. Therefore, these results indicate that upregulation of miR505 promotes apoptosis in EC by upregulating Bax and PARP and inducing G1-S stage arrest via downregulation CDK2.

A mouse model of $\mathrm{EC}$ was established to further investigate the function of miR-505 in EC in vivo. Overexpression of miR-505 significantly inhibited tumor xenograft growth and led to a reduced tumor volume. IHC staining confirmed that the tumors formed by miR-505transfected cells expressed lower levels of TGF- $\alpha$. These results provide further confirmation that miR-505 might act as tumor suppressor in EC by targeting TGFA.

\section{Conclusion}

In conclusion, we demonstrate that miR-505 suppresses cell proliferation, invasion and metastasis in EC by targeting TGFA. This knowledge may provide the basis for further investigations to identify novel diagnostic and therapeutic methods for EC.

\section{Methods}

\section{Tissue specimen collection}

67 Endometrial adenocarcinomas (ECs) and 20 normal endometrial specimens were collected from patients who underwent surgical resection at the Gynecology Department, First Affiliated Hospital of China Medical University (Shenyang, Liaoning Province, China) between May 2013 and May 2015. No patients had received any treatment before surgery. Tumor staging and pathology were based on the FIGO criteria (2009). The China Medical University ethic committee approved this research project and informed consent was obtained from each patient. Tissues were immediately frozen in liquid nitrogen and stored at $-80{ }^{\circ} \mathrm{C}$ until analysis.

\section{Cell culture and transfection}

The human endometrial cancer cell lines HEC-1B (poorly differentiated, estrogen receptor-negative endometrial adenocarcinoma) and Ishikawa (highly differentiated, estrogen receptor-positive endometrial adenocarcinoma) were obtained from the ATCC (Manassas, VA, USA) and maintained in Dulbecco's Modified Eagle's Medium (DMEM) containing $10 \%$ fetal bovine serum and $1 \%$ penicillin and streptomycin at $37{ }^{\circ} \mathrm{C}$ in a humidified atmosphere containing $5 \% \mathrm{CO}_{2}$. Cells were each transfected with miR-505 mimic using Lipofectamine 2000 reagent (Invitrogen, Carlsbad, USA) according to the manufacturer's protocol. The miR-505 mimic sequence was GGG AGC CAG GAA GUA UUG AUG U, and the scrambled mimic (Mock) sequence was ACU ACU GAG UGA CAG UAG A. The scrambled mimic was also transfected in cells as negative control.

\section{Cell proliferation assay}

Cell proliferation was monitored using CCK-8 reagent (Dojindo, Tokyo, Japan) following the manufacturer's protocol. EC cells were transfected with miR-505 mimic or scrambled mimic, seeded into 96 -well plates $\left(2 \times 10^{3}\right.$ cells per well) and incubated until adherent. Cell proliferation was assessed every $24 \mathrm{~h}$ (up to $72 \mathrm{~h}$ ); $10 \mu \mathrm{L}$ CCK- 8 solution was added to the medium in each well, 

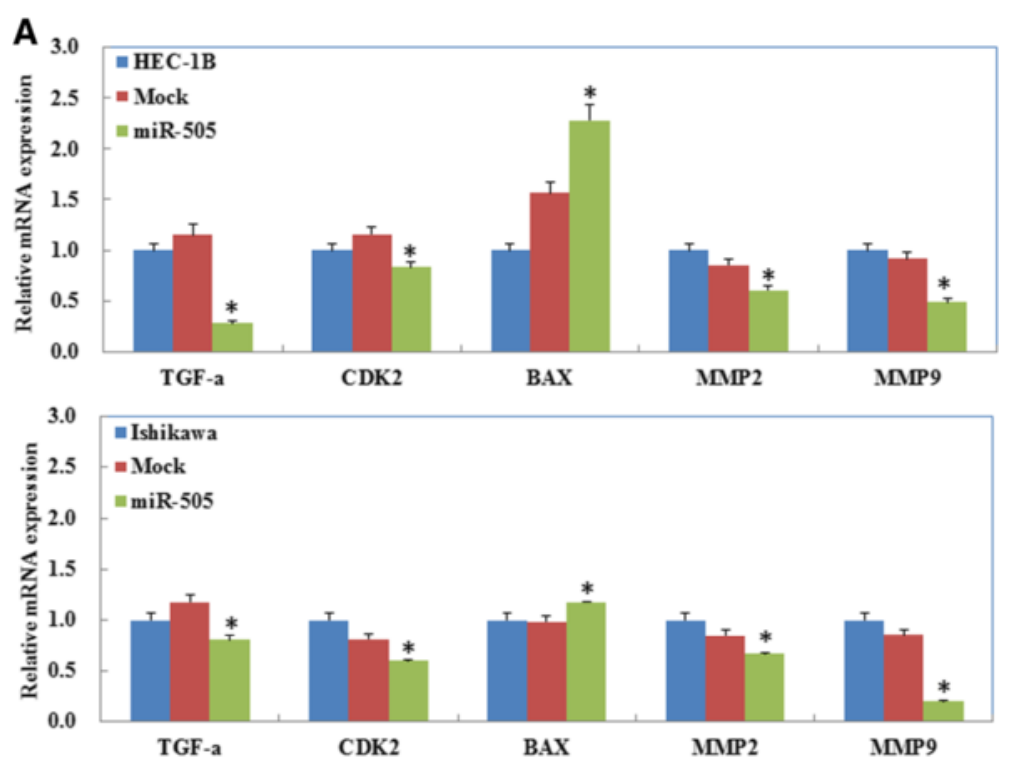

B

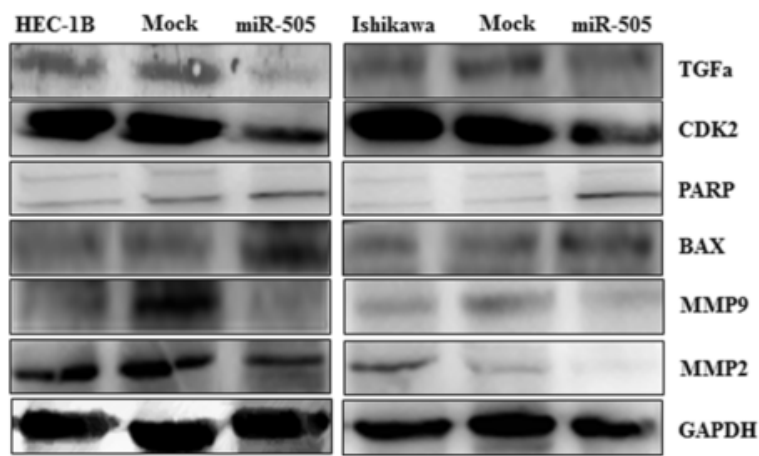

Fig. 6 Effects of miR-505 transfection on endometrial adenocarcinoma cell genotype in vitro. miR-505 overexpression reduced TGF-a, CDK2, MMP2 and MMP9 expression, while increased cleaved PARP and Bax mRNA (a) or protein (b) expression. ${ }^{*} P<0.05$

incubated for $4 \mathrm{~h}$ and the absorbance values were measured at $450 \mathrm{~nm}$ using a microplate reader.

\section{Cell cycle analysis}

Cells were trypsinized, harvested, washed twice with PBS, fixed with $70 \%$ ice-cold ethanol in $-20{ }^{\circ} \mathrm{C}$ overnight, washed with PBS, and then incubated with RNAase and stained with PI (BD Biosciences, New Jersey, USA) following the manufacturer's protocol. The PI signal was examined by a flow cytometry; a total of 10,000 cells were assessed for each sample.

\section{Apoptosis assay}

Apoptosis was quantified using 7AAD and PE-labeled Annexin V (BD Biosciences) following the manufacturer's protocol and flow cytometry. Cells were collected $48 \mathrm{~h}$ after transfection, washed twice with cold PBS, and resuspended at $1 \times 10^{6}$ cells $/ \mathrm{mL}$ and mixed with $100 \mu \mathrm{L}$ of $1 \times$ buffer and $5 \mu \mathrm{L}$ Annexin V- PE and 7AAD, incubated for
15 min in the dark, $400 \mu \mathrm{L} 1 \times$ buffer was added, and the cells were subjected to cytometry flow within $1 \mathrm{~h}$.

\section{Wound healing assay}

Cells were seeded into 6-well plates, allowed to adhere and transfected with miR-505 mimic or scrambled mimic, linear wounds were created in the confluent monolayers using a pipette tip. Cells were washed three times with PBS, the medium was changed to FBS-free DMEM, and photos were captured using a light microscope at $0 \mathrm{~h}$, $24 \mathrm{~h}$ and $48 \mathrm{~h}$. The wound healing rate $=$ (Area of original wound - Area of actual wound at different times)/Area of original wound $\times 100 \%$.

\section{In vitro cell invasion assay}

Cells transfected with miR-505 mimic or mock mimic were collected after $48 \mathrm{~h}$, re-suspended in serum-free DMEM medium, and $5 \times 10^{4}$ cells in serum-free medium were seeded into the upper chamber of Transwell inserts that had been pre-coated with Matrigel $(40 \mu \mathrm{L}$ of $8 \mathrm{mg} / \mathrm{mL}$ 


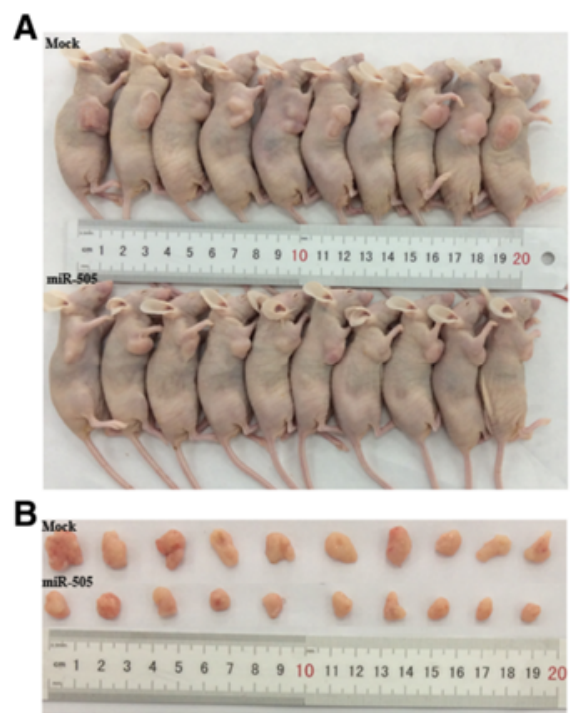

C

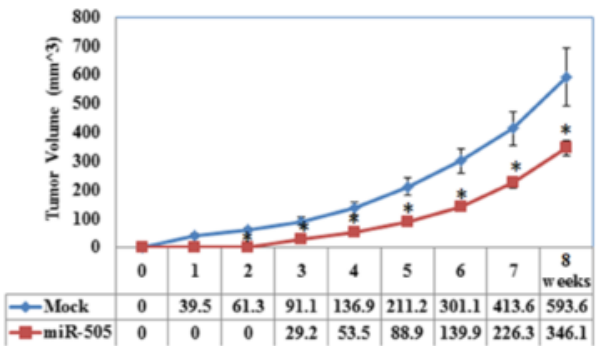

Fig. 7 Overexpression of miR-505 inhibits the growth of tumor xenografts in a mouse model. Tumor xenograft volume in nude mice treated with miR-505 was smaller than that in mock nude mice (a and $\mathbf{b})$. The growth rate was also slower than that in the mock group (c). ${ }^{*} P<0.05$

stock solution; Becton-Dickinson Labware, Bedford, MA, USA); normal culture medium was placed into the lower chamber. Cells were allowed to migrate for $48 \mathrm{~h}$ at $37^{\circ} \mathrm{C}$, then the membranes were fixed with methanol and stained with $0.1 \%$ crystal violent. Cell invasion was measured by counting the number of cells attached to the lower side of the membrane in ten high-powered (200x) fields under a light microscope.

\section{Quantitative RT-PCR}

Total RNA was isolated from the treated cells and human tissues using TRIzol reagent (Takara, Shiga, Japan)

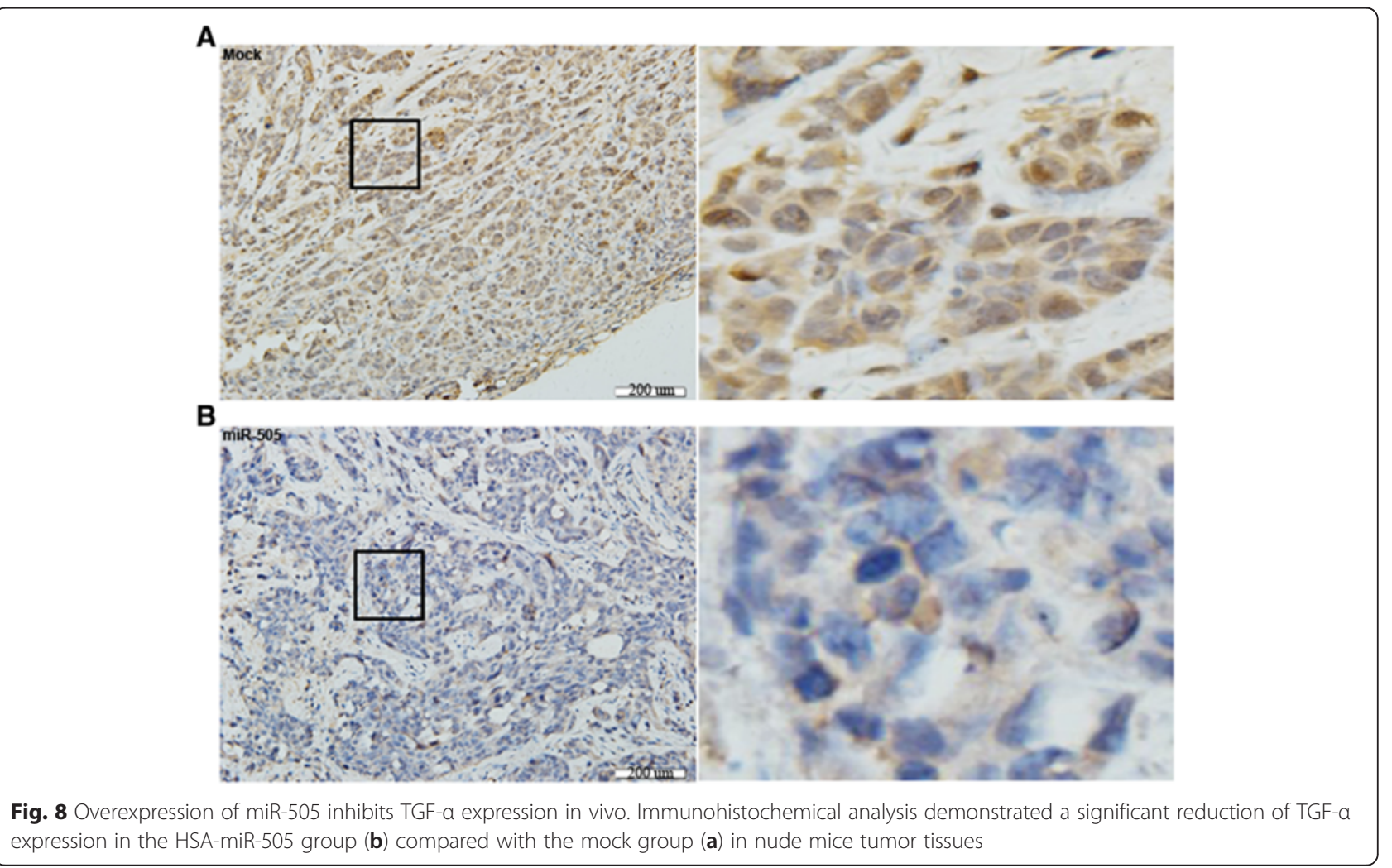


according to the manufacturer's instructions, after which OD260/280/320 was measured by spectrophotometer (Unico, Shanghai, China). The value of OD260/280 within 1.8-2.0 means high RNA quality, and the sample concentration $=$ sample dilution factor * $($ OD260-OD320 $)$ " 0.04 . $2 \mu \mathrm{g}$ was reverse-transcribed to complementary DNA (cDNA) using avian myeloblastosis virus transcriptase and random primers (Takara). Real-time RT-PCR analysis was performed in triplicate on the $\mathrm{ABI}$ prism 7000 sequence detection system (Applied Biosystems) using the SYBR Green PCR Master Mix (Applied Biosystems, Eugene, OR, USA). The relative expression of target genes was determined by comparing the threshold cycle $(\mathrm{Ct})$ of the target genes to the $\mathrm{Ct}$ of $18 \mathrm{~S}$ rRNA $(18 \mathrm{~s})$ using the $2^{-\Delta \Delta \mathrm{CT}}$ method [46]. Hairpin-it ${ }^{\text {tm }}$ microRNA and U6 snRNA Normalization RT-PCR Quantitation (GenePharma) was used to check mature miR-505 in cells.

\section{Western blotting}

Cells and tissues were lysed in ice-cold RIPA lysis buffer and the concentrations were measured using the protein assay kit (Bio-Rad Laboratories, Hercules, CA, USA). Protein samples $(60 \mu \mathrm{g})$ were separated by SDS-PAGE on $10 \%$ gels and transferred onto Hybond membranes (Amersham, Munich, Germany). After blocking with $5 \%$ fat-free milk for $1 \mathrm{~h}$, the membranes were incubated with primary antibodies against MMP2, MMP9, Cyclin A1, CDK2, Bax, PARP, TGF- $\alpha$ (1:500, Proteintech, Proteintech Group, USA) and GAPDH (1:2000, Proteintech, Proteintech Group, USA) overnight in $4{ }^{\circ} \mathrm{C}$, then incubated with anti-mouse, anti-rabbit or anti-goat IgG secondary antibodies (1:5000; Dako, Carpinteria, CA, USA) for $1 \mathrm{~h}$. The bands were developed using ECL Plus detection reagent (Santa Cruz Biotechnology) and visualized on X-ray film (Fujifilm, Tokyo, Japan) using Image Quant LAS 4000 (Fujifilm). Band densities were determined and expressed relative to the internal control GAPDH.

\section{Dual-luciferase reporter assay}

Luciferase assays were carried out to confirm the interaction between miR-505 and the 3' untranslated region (UTR) of TGFA. HEK293T cells were seeded into 24-well plates, cotransfected with $50 \mathrm{nM}$ miR-505 or scrambled mimic and $600 \mathrm{ng}$ of dual luciferase vector expressing the wild-type or mutant 3'-UTR TGF- $\alpha$ sequences. After $24 \mathrm{~h}$, the luciferase activities were measured using the DualLuciferase Reporter Assay System (Promega, Madison, WI, USA) according to the manufacturer's protocol. The ratio of firefly to renilla luciferase signal was used to normalize firefly activity for intra-experimental transfection efficiency.

\section{In vivo tumorigenesis model}

An in vivo model of EC was established by subcutaneously injecting 5-week-old female BALB/c nude mice with $1 \times 10^{7}$ HEC-1B cells transfected with miR-505 (or mock transfected) suspended in PBS (10 mice per group). The weight of the mice and tumor volumes were determined every 3 days; tumor volume was assessed by measuring the length $(L)$ and width $(W)$ using calipers (tumor volume, $\mathrm{mm}^{3}=0.5 \times \mathrm{L} \times \mathrm{W}^{2}$. After 8 weeks, the mice were euthanized and the tumors were excised, measured and photographed. All mice were obtained from Shanghai SLAC Laboratory Animal, Co., Ltd. (Shanghai, China) and housed in a specific pathogen-free environment. This experiment was in accordance with the National Institutes of Health Guide for the Care and Use of Laboratory Animals and approved by China Medical University Animal Care and Use Committee.

\section{Immunohistochemistry}

Consecutive tissue sections of the xenograft tumors were subjected to IHC to detect TGF- $\alpha$ expression. After deparaffinization, dehydration and boiling to unmask antigens, sections were blocked and incubated with TGF- $\alpha$ antibodies overnight at $4{ }^{\circ} \mathrm{C}$, then incubated with HRPconjugated anti-rabbit antibodies (Dako) for $15 \mathrm{~min}$.

\section{Statistical analysis}

Data are presented as mean $\pm \mathrm{SD}$ and were analyzed using SPSS 17.0 software (SPSS Inc., Chicago, IL, USA). The unpaired two-tailed Student's $t$-test, Mann-Whitney $U$-test and Spearman's correlation test were used to compare the two groups. All $P$-values are two-sided; $P<0.05$ represents statistical significance.

\section{Additional file}

Additional file 1: Table S1. miR-505 expression in normal endometrial and endometrial. Table S2. Correlation of miR-505 expression with different clinicopathological features of endometrial carcinoma. Table S3. Cell phenomenon studies. (DOCX $24 \mathrm{~kb}$ )

\section{Competing interests}

The authors declare that they have no competing interests.

\section{Authors' contributions}

YZ conceived the study, and analyzed interpretation. SC, K-XS, B-LL, and $\mathrm{Z}-\mathrm{HZ}$ carried out the experiments and analyzed the data. $\mathrm{Z}-\mathrm{HZ}$ gave many good suggestions about data processing and manuscript, SC wrote the first and final draft of the manuscript. All authors read and approved the final manuscript.

\section{Acknowledgements}

This work was supported by the Natural Scientific Foundation of China (Nos. 81202049; 81472440; 81472502) and Liaoning Science and Technology Grant (L2013021077).

\section{Author details}

'Department of Gynecology, The First Affiliated Hospital of China Medical University, Shenyang 110001, China. ${ }^{2}$ Department of Biochemistry and Molecular Biology, College of Basic Medicine, China Medical University, Shenyang 100013, China. 


\section{Received: 21 July 2015 Accepted: 27 January 2016}

\section{Published online: 02 February 2016}

\section{References}

1. Siegel R, Naishadham D, Jemal A. Cancer statistics, 2012. CA Cancer J Clin. 2012;62(1):10-29.

2. Deligdich L, Holika CF. Endometrial carcinoma: two diseases? Cancer Detect Prev. 1987;10(3-4):237-46.

3. Deligdisch L. When cancer of the endometrium is not a good cancer. Rev Fr Gynecol Obstet. 1990;85(10):513-6.

4. Kreizman-Shefer H, Pricop J, Goldman S, Elmalah I, Shalev E. Distribution of estrogen and progesterone receptors isoforms in endometrial cancer. Diagn Pathol. 2014:9:77.

5. Ray M, Fleming G. Management of advanced-stage and recurrent endometrial cancer. Semin Oncol. 2009;36(2):145-54.

6. Lotocki RJ, Copeland LJ, DePetrillo AD, Muirhead W. Stage I endometrial adenocarcinoma: treatment results in 835 patients. Am J Obstet Gynecol. 1983;146:141-5.

7. Boren T, Xiong Y, Hakam A, Wenham R, Apte S, Wei Z, et al. MicroRNAs and their target messenger RNAs associated with endometrial carcinogenesis. Gynecol Oncol. 2008;110(2):206-15.

8. Yoon A, Park JY, Park JY, Lee YY, Kim TJ, Choi CH, et al. Prognostic factors and outcomes in endometrial stromal sarcoma with the 2009 FIGO staging system: a multicenter review of 114 cases. Gynecol Oncol. 2014;132(1):70-5.

9. Lujambio A, Lowe SW. The microcosmos of cancer. Nature. 2012;482(7385):347-55.

10. Olivieri F, Rippo MR, Monsurrò V, Salvioli S, Capri M, Procopio AD, et al. MicroRNAs linking inflamm-aging, cellular senescence and cancer. Ageing Res Rev. 2013;12(4):1056-68.

11. Croce CM, Calin GA. miRNAs, cancer, and stem cell division. Cell. 2005;122(1):6-7.

12. Bray F, Loos AH, Oostindier M, Weiderpass E. Geographic and temporal variations in cancer of the corpus uteri: incidence and mortality in pre- and postmenopausal women in Europe. Int J Cancer. 2005;117:123-31.

13. Krek A, Grun D, Poy MN, Wolf R, Rosenberg L, Epstein EJ, et al. Combinatorial microRNA target predictions. Nat Genet. 2005;37:495-500.

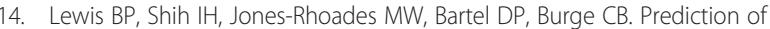
mammalian microRNA targets. Cell. 2003;115:787-98.

15. Yan J, Gumireddy K, Li A, Huang Q. Regulation of Mesenchymal Phenotype by MicroRNAs in Cancer. Curr Cancer Drug Targets. 2013;13(9):930-4.

16. Zhong $X$, Coukos G, Zhang L. miRNAs in human cancer. Methods Mol Biol. 2012;822:295-306.

17. Vasudevan S, Tong Y, Steitz JA. Switching from Repression to Activation: MicroRNAs Can Up-Regulate Translation. Science. 2007;318(5858):1931-4.

18. Yamamoto Y, Yoshioka Y, Minoura K, Takahashi RU, Takeshita F, Taya T, et al. An integrative genomic analysis revealed the relevance of microRNA and gene expression for drug-resistance in human breast cancer cells. Mol Cancer. 2011;10:135

19. Verduci L, Simili M, Rizzo M, Mercatanti A, Evangelista M, Mariani L, et al. MicroRNA (miRNA)-mediated interaction between leukemia/lymphomarelated factor (LRF) and alternative splicing factor/splicing factor 2 (ASF/SF2) affects mouse embryonic fibroblast senescence and apoptosis. J Biol Chem. 2010;285:39551-63.

20. Zhu M, Yi M, Kim CH, Deng C, Li Y, Medina D, et al. Integrated miRNA and mRNA expression profiling of mouse mammary tumor models identifies miRNA signatures associated with mammary tumor lineage. Genome Biol. 2011;12(8):R77.

21. Esquela-Kerscher A, Slack FJ. Oncomirs - microRNAs with a role in cancer. Nat Rev Cancer. 2006;6(4):259-69.

22. Qin Z, He W, Tang J, Ye Q, Dang W, Lu Y, et al. MicroRNAs Provide Feedback Regulation of Epithelial-Mesenchymal Transition Induced by Growth Factors. J Cell Physiol. 2015; May 29.

23. Jonsdottir K, Janssen SR, Da Rosa FC, Gudlaugsson E, Skaland I, Baak JP, et al. Validation of Expression Patterns for Nine miRNAs in 204 Lymph-Node Negative Breast Cancers. PLoS One. 2012;7(11), e48692.

24. Badawy AA, El-Hindawi A, Hammam O, Moussa M, Gabal S, Said N. Impact of epidermal growth factor receptor and transforming growth factor-a on hepatitis C virus-induced hepatocarcinogenesis. APMIS. 2015;123(10):823-31.

25. Giricz O, Calvo V, Peterson EA. TACE-dependent TGF-alpha shedding drives triple-negative breast cancer cell invasion. Int J Cancer. 2013;133(11):2587-95.
26. DeHaan AM, Wolters NM, Keller ET and Ignatoski KM. EGFR ligand switch in late stage prostate cancer contributes to changes in cell signaling and bone remodeling. Prostate. 2009; 69:528-37.

27. Ferrer I, Alcántara S, Ballabriga J, Olivé M, Blanco R, Rivera R, et al. Transforming growth factor-a (TGF-a) and epidermal growth factor-receptor (EGF-R) immunoreactivity in normal and pathologic brain. Prog Neurobiol. 1996;49(2):99-123.

28. Schreiber AB, Winkler ME, Derynck R. Transforming growth factoralpha:a more potent angiogenic mediator than epidermal growth factor. Science. 1986;232:1250-3.

29. Zhu C, Li J, Ding Q, Cheng G, Zhou H, Tao L, et al. miR-152 controls migration and invasive potential by targeting TGFa in prostate cancer cell lines. Prostate. 2013;73(10):1082-9.

30. Jin Y, Peng D, Shen Y, Xu M, Liang Y, Xiao B, et al. MicroRNA-376c inhibits cell proliferation and invasion in osteosarcoma by targeting to transforming growth factor-alpha. DNA Cell Biol. 2013;32(6):302-9.

31. Qin W, Pan Y, Zheng X, Li D, Bu J, Xu C, et al. MicroRNA-124 regulates TGF-a-induced epithelial-mesenchymal transition in human prostate cancer cells. Int J Oncol. 2014;45(3):1225-31.

32. Hou CH, Lin FL, Tong KB, Hou SM, Liu JF. Transforming growth factor alpha promotes osteosarcoma metastasis by ICAM-1 and PI3K/Akt signaling pathway. Biochem Pharmacol. 2014;89(4):453-63.

33. Kimura M, Moteki H, Ogihara M. Involvement of endogenous transforming growth factor-a in signal transduction pathway for interleukin-1 $\beta$-induced hepatocyte proliferation. Eur J Pharmacol. 2014;745:223-33.

34. Mouradian M, Kikawa KD, Johnson ED, Beck KL, Pardini RS. Key roles for GRB2-associated-binding protein 1, phosphatidylinositol-3-kinase, cyclooxygenase 2, prostaglandin E2 and transforming growth factor alpha in linoleic acid-induced upregulation of lung and breast cancer cell growth. Prostaglandins Leukot Essent Fatty Acids. 2014;90(4):105-15.

35. Troiani T, Martinelli E, Napolitano S, Vitagliano D, Ciuffreda LP, Costantino S, et al. Increased TGF-a as a Mechanism of Acquired Resistance to the AntiEGFR Inhibitor Cetuximab through EGFR-MET Interaction and Activation of MET Signaling in Colon Cancer Cells. Clin Cancer Res. 2013;19(24):6751-65.

36. Tabernero J, Cervantes A, Rivera F, Martinelli E, Rojo F, von Heydebreck A, et al. Pharmacogenomic and pharmacoproteomic studies of cetuximab in metastatic colorectal cancer: biomarker analysis of a phase I dose-escalation study. J Clin Oncol. 2010;28:1181-9.

37. Shih IM, Herlyn M. Autocrine and paracrine roles for growth factors in melanoma. In Vivo. 1994;8(1):113-23.

38. Tarhini AA, Lin Y, Yeku O, LaFramboise WA, Ashraf M, Sander C, et al. A fourmarker signature of TNF-RII, TGF-a, TIMP-1 and CRP is prognostic of worse survival in high-risk surgically resected melanoma. J Transl Med. 2014;12:19.

39. Mclnnes C, Wang J, Al Moustafa AE, Yansouni C, O'Connor-McCourt M, Sykes BD. Structure-based minimization of transforming growth factor-alpha (TGFalpha) through NMR analysis of the receptor-bound ligand. Design, solution structure, and activity of TGF-alpha 8-50. J Biol Chem. 1998;273(42):27357-63.

40. Somerville RP, Oblander SA, Apte SS. Matrix metalloproteinases: old dogs with new tricks. Genome Biol. 2003;4(6):216.

41. Sherr CJ, Roberts JM. Living with or without cyclins and cyclin-dependent kinases. Genes Dev. 2004;18:2699-711.

42. Shames DS, Girard L, Gao B, Sato M, Lewis CM, Shivapurkar N, et al. A genomewide screen for promoter methylation in lung cancer identifies novel methylation markers for multiple malignancies. PLoS Med. 2006;3(12), e486.

43. Volkmann N, Marassi FM, Newmeyer DD, Hanein D. The rheostat in the membrane: BCL-2 family proteins and apoptosis. Cell Death Differ. 2014;21(2):206-15.

44. Jeggo PA. DNA repair: PARP - another guardian angel? Curr Biol. 1998;8(2): R49-51.

45. Donizy P, Halon A, Surowiak P, Pietrzyk G, Kozyra C, Matkowski R. Correlation between PARP-1 immunoreactivity and cytomorphological features of parthanatos, a specific cellular death in breast cancer cells. Eur J Histochem. 2013;57(4), e35.

46. Chen S, Jiao JW, Sun KX, Zong ZH, Zhao Y. MicroRNA-133b targets glutathione $\mathrm{S}$-transferase $\pi$ expression to increase ovarian cancer cell sensitivity to chemotherapy drugs. Drug Des Devel Ther. 2015;9:5225-35. 